\title{
Modular symmetry and temperature flow of conductivities in quantum Hall systems with varying Zeeman energy
}

\author{
Brian P. Dolan* \\ Department of Mathematical Physics, National University of Ireland, Maynooth, Ireland \\ and Dublin Institute for Advanced Studies, 10 Burlington Road, Dublin, Ireland
}

(Received 23 September 2010; published 17 November 2010)

\begin{abstract}
The behavior of the critical point between quantum Hall plateaux, as the Zeeman energy is varied, is analyzed using modular symmetry of the Hall conductivities following from the law of corresponding states. Flow diagrams for the conductivities as a function of temperature, with the magnetic field fixed, are constructed for different Zeeman energies, for samples with particle-hole symmetry.
\end{abstract}

DOI: 10.1103/PhysRevB.82.195319

PACS number(s): 73.43.Nq, 05.30.Fk, 05.30.Rt, 02.20.-a

\section{INTRODUCTION}

The quantum Hall effect continuous to intrigue both experimentalists and theorists not only because of the beautifully rich patterns visible in the data but also because of the fascinating physics involved in the collective phenomena of strongly interacting systems. The first suggestion of a connection between the quantum Hall effect and the modular group appeared in Ref. 1, although these authors focused on a subgroup of the full modular group that did not turn out to have any direct relevance to the current experimental data. Subsequent papers on symmetries of the phase diagram of the quantum Hall effect ${ }^{2,3}$ appeared almost simultaneously from two very different directions and laid the foundations for the application of modular symmetry to the quantum Hall effect. Although Ref. 2 did not use the mathematical language of modular symmetry the "Law of Corresponding States" put forward in that reference is in fact equivalent to the assumption of modular symmetry. ${ }^{4}$

Modular symmetry gives predictions ${ }^{5}$ for the manner in which the conductivity of a two-dimensional quantum Hall sample flows, as the temperature is varied keeping the magnetic field fixed-predictions which have already received strong experimental support. ${ }^{6-10}$

While the flow diagram presented in Ref. 5 was for spinsplit samples the experimental data presented in Ref. 6 is for spin-degenerate samples. Zeeman splitting in the context of modular symmetry was analyzed in Ref. 11 and a flow diagram, deformed by interactions between adjacent Landau levels, was given but the fully spin-degenerate case has not yet been treated using modular symmetry

In this paper restrictions on the temperature flow due to modular symmetry are combined with a Zeeman splitting analysis to determine the way in which temperature flow changes as the Zeeman splitting is smoothly varied between the two extremes of samples with well-split spins and fully spin-degenerate samples. The analysis is restricted to situations with particle-hole symmetry, as modular symmetry is particularly powerful in this case but does not give strong predictions otherwise. A central prediction of the analysis is that pairs of critical points of the quantum Hall phase transitions between adjacent plateaux in spin-split samples must merge as the Zeeman splitting is reduced, as shown in Figs. 12 and 13.

\section{MODULAR GROUP}

The law of corresponding states, 2,12 for isotropic quantum Hall samples with spins well split, can be written in terms of the complex conductivity

$$
\sigma=\sigma_{x y}+i \sigma_{x x}
$$

(for isotropic samples $\sigma_{x x}=\sigma_{y y}$ ). A general map between two quantum Hall states can be constructed by iterating two generating maps: $:^{3,4,13}$ the Landau level addition transformation, L,

$$
\sigma \rightarrow \sigma+1
$$

and the flux attachment transformation, $\mathbf{F}^{2}$,

$$
\sigma \rightarrow \frac{\sigma}{2 \sigma+1}
$$

which attaches two units of statistical gauge field flux to each electron (we use units with $\frac{e^{2}}{h}=1$ ).

In samples which enjoy particle-hole symmetry there is a third map, the particle-hole transformation, $\mathbf{P}_{1}$,

$$
\sigma \rightarrow 1-\bar{\sigma} \text {. }
$$

These maps generalize Jain's transformations on groundstate wave functions ${ }^{14}$ to include nonzero Ohmic conductivity. Repeated iteration of $\mathbf{F}^{2}$ and $\mathbf{L}$ generate an infinite discrete group which we shall denote $\Gamma_{0}(2)$.

The infinite discrete group generated by repeated applications of $\mathbf{L}$ and $\mathbf{F}$ is called the modular group and is usually denoted by $\Gamma(1)$ in the mathematical literature. ${ }^{15}$ A general element $\gamma \in \Gamma(1)$ sends

$$
\sigma \rightarrow \gamma(\sigma):=\frac{a \sigma+b}{c \sigma+d},
$$

where $a, b, c$, and $d$ are any four integers satisfying $a d-b c=1$. Group multiplication can be realized in terms of the $2 \times 2$ matrix

$$
\gamma=\left(\begin{array}{ll}
a & b \\
c & d
\end{array}\right)
$$

and demanding that det $\gamma=1$. It is easy to check from the definition [Eq. (1)] that, for any three such matrices satisfying $\gamma_{1} \gamma_{2}=\gamma_{3}$, we have $\gamma_{1}\left[\gamma_{2}(\sigma)\right]=\gamma_{3}(\sigma)$. Thus the group 
multiplication law is given by matrix multiplication.

The full modular group $\Gamma(1)$ is not a symmetry of quantum Hall effect, for example, the element $\left(\begin{array}{cc}0 & 1 \\ -1 & 0\end{array}\right)$ sends $\sigma \rightarrow-1 / \sigma$ which has a fixed point for $\sigma=i$, i.e., $\sigma_{x x}=1$ and $\sigma_{x y}=0$. There is no indication in the experimental data on the quantum Hall effect that this point has any special significance (though it is important in the insulatorsuperconductor phase transition ${ }^{16,17}$ ).

The group $\Gamma_{0}(2)$ is a subgroup of the modular group, it is represented by matrices of the form [Eq. (2)] with the extra condition that $c$ be even. In matrix notation Landau level addition and flux attachment are represented by $\mathbf{L}=\left(\begin{array}{ll}1 & 1 \\ 0 & 1\end{array}\right)$ and $\mathbf{F}^{2}=\left(\begin{array}{ll}1 & 0 \\ 2 & 1\end{array}\right)$.

If the electron spins are not split then the story is different. For simplicity first consider the situation if Zeeman splitting is completely absent and states corresponding to two different electron spins are completely degenerate. Then Landau level addition sends

$$
\sigma \rightarrow \sigma+2
$$

or $\frac{\sigma}{2} \rightarrow \frac{\sigma}{2}+1$, which is $\mathbf{L}^{2}=\left(\begin{array}{ll}1 & 2 \\ 0 & 1\end{array}\right)$. Attaching two units of flux to each electron sends

$$
\frac{\sigma}{2} \rightarrow \frac{\left(\frac{\sigma}{2}\right)}{2\left(\frac{\sigma}{2}\right)+1} \Rightarrow \sigma \rightarrow \frac{\sigma}{\sigma+1},
$$

which is $\mathbf{F}=\left(\begin{array}{ll}1 & 0 \\ 1 & 1\end{array}\right)$ acting on $\sigma$. Thus spin generate Landau levels give rise to an infinite discrete group generated by $\mathbf{L}^{2}$ and F. A general element of this group is of the form $\gamma=\left(\begin{array}{ll}a & b \\ c & d\end{array}\right)$ with $a, b, c$, and $d$ integers satisfying $a d-b c=1$ but with $b$ restricted to be even. We denote this group by $\Gamma^{0}(2)$. In fact that $\Gamma^{0}(2)$ acting on $\sigma$ is the same as $\Gamma_{0}(2)$ acting on $\frac{\sigma}{2}$. Particle-hole symmetry for degenerate spins is also modified to $\mathbf{P}_{2}=\sigma \rightarrow 2-\bar{\sigma}$, so $\mathbf{P}_{2}=\mathbf{P}_{1}+1$.

For spins that have a slight splitting so that the Landau levels corresponding to opposite spins are not completely degenerate but are still close enough for there to be some mixing between Landau level wave functions, one expects $\mathbf{L}^{2}$ to be a symmetry rather than $\mathbf{L}$. At the same time adding two units of statistical flux to each electron in the individual levels is described by $\mathbf{F}^{2}$. For this intermediate case therefore the group is generated by $\mathbf{L}^{2}$ and $\mathbf{F}^{2}$ and a general element is represented by $\gamma=\left(\begin{array}{ll}a & b \\ c & d\end{array}\right)$ with $a d-b c=1$ and both $b$ and $c$ even integers. This group is denoted by $\Gamma(2)$. Clearly $\Gamma(2) \subset \Gamma_{0}(2)$ and $\Gamma(2) \subset \Gamma^{0}(2)$. We are led to suggest the following sequence of symmetries as the Zeeman splitting relative to the cyclotron energy is varied from large to small:

$$
\Gamma_{0}(2) \rightarrow \Gamma(2) \rightarrow \Gamma^{0}(2),
$$

thus the symmetry first decreases and then increases again as the Zeeman splitting is varied.

Since the modular group has an infinite number of elements the law of corresponding states maps between an infinite number of quantum Hall phases, which is clearly a mathematical idealization which is never realized in any physical system. Obviously the symmetry breaks down in various limits, such as weak magnetic field, when the Landau level splitting becomes comparable with thermal energies, or very strong magnetic fields, when a Wigner crystal is expected to form. The range of validity is discussed in Ref. 18.

It was pointed out in Ref. 19 that the law of corresponding states applies to the ac conductivity in the limit of infinite frequency rather than the dc conductivity. This is because the conductivity is a function of frequency over temperature, $\sigma_{x x}\left(\frac{\omega}{T}\right)$, and the derivation of the law of corresponding states in Ref. 2 takes the limit $T \rightarrow 0$ before $\omega \rightarrow 0$ and this does not in general commute with the limit required to extract the dc conductivity, namely, $\omega \rightarrow 0$ before $T \rightarrow 0$. The precise relationship between these two limits can only be explored in the context of a specific microscopic theory for the conductivity and is not accessible solely through infrared effective action techniques such as the law of corresponding states. Nevertheless the law of corresponding states has been applied to dc conductivities, for example, with regard to temperature flows, and the experimental data are in remarkable agreement with the predictions. ${ }^{6-10,18}$ While some microscopic models may display a symmetry which makes the order in which the limits are taken irrelevant ${ }^{19}$ this is not generic but since we do not commit ourselves to a specific model here we cannot address this question directly. Rather our philosophy will be to develop the predictions of the law of corresponding states, see where they lead, and future experiments will test their validity.

\section{TEMPERATURE FLOW}

Scaling arguments ${ }^{20}$ suggest that, at low temperatures, the dc conductivity should be a function of a single variable, $\sigma\left(\frac{\Delta B}{T^{\kappa}}\right)$, rather than of the temperature $T$ and magnetic field $B$ separately. Here $\Delta B=B-B_{c}$ is the deviation of the magnetic field from the critical value $B_{c}$ separating two quantum Hall phases and $\kappa$ is a scaling exponent, experimentally $\kappa \approx 0.42 \pm 0.01 .^{21}$ The conductivity depends on the electron scattering length $l$ and we define the scaling function

$$
\Sigma_{l}(\sigma, \bar{\sigma})=-l \frac{d \sigma}{d l} .
$$

Assuming $l$ is a strictly monotonic function of temperature, increasing as $T$ decreases, the temperature flow described by the scaling function

$$
\Sigma_{T}(\sigma, \bar{\sigma})=T \frac{d \sigma}{d T}
$$

will have the same topology as the flow described by $\Sigma_{l}$-they will have the same fixed points, $\Sigma_{T}=0$ if and only if $\Sigma_{l}=0$. We do not need to determine either $\Sigma_{l}$ or $\Sigma_{T}$ exactly, in fact if $s(T)$ is any monotonic function of $T$, decreasing as $T$ decreases, then the flow described by

$$
\Sigma_{s}(\sigma, \bar{\sigma})=s \frac{d \sigma}{d s}
$$

will have the same topology as both that of $\Sigma_{T}$ and of $\Sigma_{l}$.

If the law of corresponding states correctly describes the low-temperature physics then the scaling flow commutes 
with the law of corresponding states map. From this it can be concluded that any value of the complex conductivity $\sigma_{*}$ that is a fixed point of the modular group [in the sense that there exists an element $\gamma$ of the modular group such that $\left.\gamma\left(\sigma_{*}\right)=\sigma_{*}\right]$ must also be a fixed point of the scaling flow. ${ }^{4,13}$ This implies that

$$
\Sigma_{S}\left(\sigma_{*}, \bar{\sigma}_{*}\right)=0 .
$$

This follows because the assumption $\gamma\left(\sigma_{*}\right)=\sigma_{*}$ requires

$$
\begin{aligned}
\Sigma_{s}\left(\sigma_{*}, \bar{\sigma}_{*}\right) & =\Sigma_{s}\left[\gamma\left(\sigma_{*}\right), \gamma\left(\bar{\sigma}_{*}\right)\right]=\left.s \frac{d \gamma(\sigma)}{d s}\right|_{\sigma_{*}} \\
& =\frac{1}{\left(c \sigma_{*}+d\right)^{2}} \Sigma_{s}\left(\sigma_{*}, \bar{\sigma}_{*}\right),
\end{aligned}
$$

which is only possible if $\left(c \sigma_{*}+d\right)^{2}=1$ or if $\Sigma_{s}\left(\sigma_{*}, \bar{\sigma}_{*}\right)=0$ or $\infty$. If $\left(c \sigma_{*}+d\right)^{2}=1$ then $a \sigma_{*}+b= \pm \sigma_{*}$ and, excluding the trivial case $a= \pm 1, b=0$, this is not possible if the Ohmic conductivity $\left(\sigma_{*}\right)_{x x}>0$ at the fixed point. Assuming $\Sigma_{s}\left(\sigma_{*}, \bar{\sigma}_{*}\right)$ is not infinite we conclude that $\Sigma_{s}\left(\sigma_{*}, \bar{\sigma}_{*}\right)=0$ and $\sigma_{*}$ is a fixed point of the flow. The fixed points with $\operatorname{Im}\left(\sigma_{*}\right)>0$ are isolated and easy to enumerate since

$$
\gamma\left(\sigma_{*}\right)=\sigma_{*} \Rightarrow \sigma_{*}=\frac{a-d \pm \sqrt{(a+d)^{2}-4}}{2 c} .
$$

Now $a d-b c=1$, with $b c$ even for all three groups $\Gamma_{0}(2)$, $\Gamma^{0}(2)$, and $\Gamma(2)$, hence $a d$ must be odd so both $a$ and $d$ must be odd. Demanding $\operatorname{Im}\left(\sigma_{*}\right)>0$ then requires that $-2<a+d<2$. Hence $a+d=0, \pm 1$ but $a$ and $d$ are both odd so \pm 1 is ruled out and we can conclude that $d=-a$. Hence

$$
\sigma_{*}=\frac{a+i}{c}
$$

as the Ohmic conductivity, $\operatorname{Im}\left(\sigma_{*}\right)$, cannot be negative. A matrix $\gamma$ that leaves $\sigma_{*}$ fixed must now be of the form

$$
\gamma=\left(\begin{array}{cc}
a & b \\
c & -a
\end{array}\right)
$$

with $b c=-\left(1+a^{2}\right)$ with $a$ odd. Let $a=2 p+1$ for some integer $p$, then $b c=-4 p(p+1)-2$ and $b c=2 \bmod 4$. In particular, $b$ and $c$ cannot both be even so $\Gamma(2)$ has no fixed points with $\operatorname{Im}(\sigma)>0$.

To summarize: $\Gamma_{0}(2)$ has fixed points above the real axis of the form [Eq. (4)] with $a$ odd and $c$ even; $\Gamma^{0}(2)$ has fixed points above the real axis of the form [Eq. (4)] with $a$ and $c$ both odd; $\Gamma(2)$ has no fixed points above the real axis.

Although any fixed point of the modular group with $\operatorname{Im}\left(\sigma_{*}\right)>0$ must be a fixed point of the flow the converse does not necessarily hold, there could be fixed points of the flow that are not fixed points of the modular group. Any such point would have an infinite number of images under the group action. However there is no sign any such extra fixed points in the experimental data for spin-split samples so we shall assume that there are no fixed points for $\Gamma_{0}(2)$, other than those required by the symmetry. For brevity in the following this will be referred as the minimalist assumption. If we assume that the topology of the flow varies smoothly as the Zeeman energy is varied then the $\Gamma_{0}(2)$ fixed points cannot suddenly disappear when $\Gamma_{0}(2)$ is broken to the smaller group $\Gamma(2)$, they must move down toward the real axis. We shall likewise assume that the only fixed points of the flow for degenerate spins are those of $\Gamma^{0}(2)$ and these move smoothly down toward the real axis as the degeneracy is lifted.

The topology of the flow is determined by the fixed points and some other rather mild assumptions, ${ }^{5}$ such as decreasing Ohmic conductivity when $\sigma_{x x} \gg \sigma_{x y}$ as the temperature is reduced, as in a semiconductor, and attractive fixed points at integer quantum Hall plateaux. We can plot the flow by changing variables from $\sigma$ to $\lambda(\sigma)$ where $\lambda$ is invariant under $\Gamma(2)$, i.e., $\lambda[\gamma(\sigma)]=\lambda(\sigma)$, with $\gamma \in \Gamma(2) .{ }^{11,22}$ Since $\Sigma_{s}(\sigma, \bar{\sigma})$ represents a vector flow in a two-dimensional space this is just a change in coordinates in that space. In the new parametrization the flow is given by

$$
\Sigma_{\lambda}(\lambda, \bar{\lambda})=s \frac{d \lambda}{d s}=\Sigma_{s}(\sigma, \bar{\sigma}) \lambda^{\prime} \Rightarrow \Sigma_{s}(\sigma, \bar{\sigma})=\frac{\Sigma_{\lambda}}{\lambda^{\prime}},
$$

where $\lambda^{\prime}=\frac{d \lambda}{d \sigma}$.

The invariant function $\lambda$ that has the smallest number of poles and zeros in the complex plane is unique, up to a constant rescaling and addition of a constant. ${ }^{23}$ It is most easily expressed in terms of Jacobi $\vartheta$ functions,

$$
\lambda=\frac{\vartheta_{2}^{4}}{\vartheta_{3}^{4}}
$$

where

$$
\vartheta_{3}(\sigma)=\sum_{n=-\infty}^{\infty} e^{i \pi n^{2} \sigma} \quad \text { and } \quad \vartheta_{2}=2 \sum_{n=0}^{\infty} e^{i \pi(n+1 / 2)^{2} \sigma} .
$$

It is also useful to define

$$
\vartheta_{4}^{4}(\sigma)=\sum_{n=-\infty}^{\infty}(-1)^{n} e^{i \pi n^{2} \sigma}=\vartheta_{3}^{4}(\sigma)-\vartheta_{2}^{4}(\sigma) .
$$

Then these functions have the following transformation properties under $\mathbf{L}$ and $\mathbf{F}^{2}$ :

$$
\begin{gathered}
\vartheta_{2}(\sigma+1)=e^{i \pi / 4} \vartheta_{2}(\sigma), \quad \vartheta_{2}\left(\frac{\sigma}{2 \sigma+1}\right)=\sqrt{2 \sigma+1} \vartheta_{2}(\sigma), \\
\vartheta_{3}(\sigma+1)=\vartheta_{4}(\sigma), \quad \vartheta_{3}\left(\frac{\sigma}{2 \sigma+1}\right)=\sqrt{2 \sigma+1} \vartheta_{3}(\sigma), \\
\vartheta_{4}(\sigma+1)=\vartheta_{3}(\sigma), \quad \vartheta_{4}\left(\frac{\sigma}{2 \sigma+1}\right)=\sqrt{2 \sigma+1} e^{-i \pi / 2} \vartheta_{4}(\sigma),
\end{gathered}
$$

(we use the notation of Ref. 23).

Furthermore under $\mathbf{P}_{2}, \quad \vartheta_{i} \rightarrow \bar{\vartheta}_{i}$ for $i=2,3,4$. Hence particle-hole interchange swaps $\lambda \leftrightarrow \bar{\lambda}$ and assuming particlehole symmetry has the important consequence for the scaling function that 


$$
\Sigma_{\lambda}(\lambda, \bar{\lambda})=\overline{\Sigma_{\lambda}(\lambda, \bar{\lambda})}=\Sigma_{\lambda}(\bar{\lambda}, \lambda) .
$$

This then implies that an expansion of $\Sigma$ in powers of $\lambda$ and $\bar{\lambda}$ has only real coefficients and we draw the important conclusion that, starting from any point for which $\lambda$ is real, the flow can never generate an imaginary part for $\lambda$. In other words any curve on which $\lambda$ is real is in integral curve of the flow: ${ }^{22}$ this is a key observation in creating the flow diagrams below. In particular, $\lambda$ is real on vertical lines above the integer points on the real line and one the semicircles of radius $1 / 2$ joining the integers (see Fig. 1). While $\lambda$ is not invariant under the larger groups $\Gamma_{0}(2)$ and $\Gamma^{0}(2)$ the following functions of $\lambda$ are (1) $\mu=\frac{\lambda-1}{\lambda^{2}}=-\frac{\vartheta_{3}^{4} \vartheta_{4}^{4}}{\vartheta_{2}^{8}}$ is invariant under $\Gamma_{0}(2)$ and (2) $\rho=\frac{\lambda}{(1-\lambda)^{2}}=\frac{\vartheta_{2}^{4} \vartheta_{3}^{4}}{\vartheta_{4}^{8}}$ is invariant under $\Gamma^{0}(2)$.

The functions $\mu$ and $\rho$ can be used to determine the topology of the flow for $\Gamma_{0}(2)$ and $\Gamma^{0}(2)$, respectively. Fixed points of $\Gamma_{0}(2)$, respectively, $\Gamma^{0}(2)$, must be fixed points of the flow, and these are enumerated in Eq. (4). Next, as above, we argue that demanding particle-hole symmetry implies that curves on which $\mu$, respectively, $\rho$, is real will be integral curves of the flow. The flow can then be modeled qualitatively by taking $\Sigma_{s}$ to be a meromorphic function of $\sigma$, $\Sigma_{s}(\sigma)$. Although there is no physical argument for meromorphicity by plotting the flow in this case we can get a picture of what it looks like, the inclusion of $\bar{\sigma}$ dependence can only result in a smooth deformation of the meromorphic flow which leaves the fixed points invariant. Meromorphic functions satisfying Eq. (3) are called modular forms in the mathematical literature ${ }^{15}$ and their properties are well known. For $\Gamma_{0}(2)$ it was shown in ${ }^{5}$ that the minimalist assumption leads to

$$
\Sigma_{s}(\sigma)=-\frac{\mu}{\mu^{\prime}}=\frac{\lambda(1-\lambda)}{\lambda^{\prime}(2-\lambda)}=\frac{1}{i \pi\left(2 \vartheta_{3}^{4}-\vartheta_{2}^{4}\right)},
$$

where $\mu^{\prime}=\frac{d \mu}{d \sigma}$ [the second and third forms of this equation rely on Eq. (6) and the fact that

$$
\frac{\lambda(1-\lambda)}{\lambda^{\prime}}=\frac{1}{i \pi \vartheta_{3}^{4}},
$$

a result that can be derived using the techniques in Refs. 5 and 11]. The integral curves of the flow [Eq. (8)] are plotted in Fig. 3.

It should be borne in mind that Eq. (8) does not give a quantitative description of the temperature flow because the function $s(T)$ is undetermined. By changing $s(T), \Sigma_{s}$ can be multiplied by a function of $T$ but as long as $s(T)$ is strictly monotonic and real this will not change the fixed points nor will it change the fact that curves on which $\mu$ is real are integral curves of the flow-it will merely change the rate at which the flow lines in Fig. 3 are traversed as the temperature is changed, and this rate is not evident in the figure. Also physical quantum Hall samples cannot be expected to give meromorphic flow, in a real sample $\Sigma(\sigma, \bar{\sigma})$ will depend on $\bar{\sigma}$ and $\sigma$ independently, but similar arguments apply: $\bar{\sigma}$ dependence can only distort the flow smoothly from the meromorphic flow shown leaving the fixed points invariant and, again assuming particle-hole symmetry, the vertical lines above the

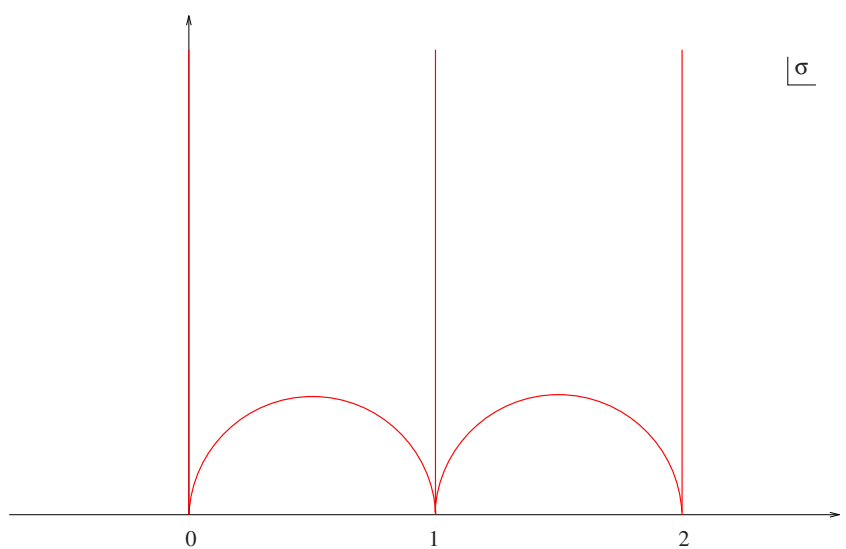

FIG. 1. (Color online) Lines on which the invariant function of $\Gamma(2), \lambda(\sigma)$ in the text, is real.

integers and the semicircles, together with their images under $\Gamma_{0}(2)$, will not be affected [Eq. (7) does not require meromorphicity]. Figure 3 should be compared to the experimental data in Refs. 7-9 for spin-split samples.

For $\Gamma^{0}(2)$ similar arguments applied to $\rho$ lead to

$$
\Sigma_{s}(\sigma)=\frac{\rho}{\rho^{\prime}}=\frac{\lambda(1-\lambda)}{\lambda^{\prime}(1+\lambda)}=\frac{1}{i \pi\left(\vartheta_{3}^{4}+\vartheta_{2}^{4}\right)}
$$

and this flow is plotted in Fig. 11. This should be compared to the experimental flow for the spin-degenerate sample in Ref. 6-the agreement is remarkable.

For samples intermediate between degenerate and wellsplit spins $\Gamma(2)$ symmetry is not as powerful as there are no fixed points with $\sigma_{x x}>0$. Nevertheless we would expect there to be fixed points of the flow, the fixed points of $\Gamma_{0}(2)$ for spin-split samples and of $\Gamma^{0}(2)$ for spin-degenerate samples can hardly just disappear when the Zeeman splitting is smoothly varied. The minimalist assumption cannot be used for $\Gamma(2)$. We can however assume that the fixed points of $\Gamma_{0}(2)$ and $\Gamma^{0}(2)$ persist when the Zeeman splitting is varied but their position is no longer dictated by modular symmetry. We seek a smooth deformation from Figs. 3-11, Eqs. (8)-(10), as the Zeeman splitting is increased, a deformation which is compatible with particle-hole symmetry, $\overline{\Sigma_{\lambda}(\lambda)}=\Sigma_{\lambda}(\bar{\lambda})$. In order to avoid creating new spurious fixed points we keep the order of the polynomials in $\lambda$ fixed in the numerators and denominators of Eqs. (8) and (10), and this dictates that the interpolating flow must be of the form

$$
\Sigma_{s}(\sigma)=\frac{\lambda(A+B \lambda)}{\lambda^{\prime}(C+D \lambda)},
$$

where $A, B, C$, and $D$ are constants. Particle-hole symmetry requires $\overline{\Sigma_{s}(\sigma)}=\Sigma_{s}(\bar{\sigma})$, which dictates that $A, B, C$, and $D$ be real. Equation (9) shows that a factor $\frac{(A+B \lambda)}{\vartheta_{3}^{4}(1-\lambda)}$ will appear in $\Sigma_{s}(\sigma)$, unless $A=-B$, and this would introduce a new zero when $\lambda=-\frac{A}{B}$ that is not there in either Eq. (8) or Eq. (10). To avoid this we set $A=-B$ and the only possible deformation that is compatible with our assumptions is, up to an overall constant factor, 


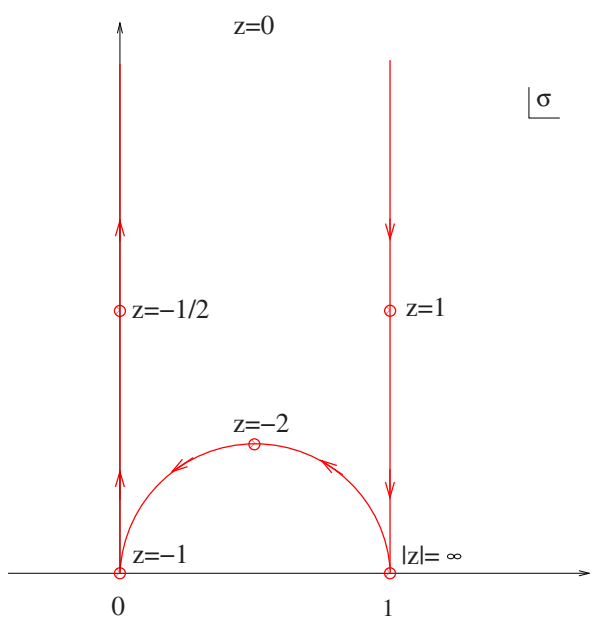

FIG. 2. (Color online) The movement of the $\Gamma_{0}(2)$ fixed $\sigma_{*}=\frac{1+i}{2}$ as the parameter $z$ is varied away from -2 . The arrows show the direction of increasing $z$.

$$
\Sigma_{s}(\sigma)=z \frac{\lambda(1-\lambda)}{\lambda^{\prime}(\lambda+z)}=\frac{1}{i \pi} \frac{z}{\left(\vartheta_{2}^{4}+z \vartheta_{3}^{4}\right)}
$$

with $z$ independent of $\sigma$ and real. The free parameter $z$ varies from $z=-2$ for $\Gamma_{0}(2)$ to $z=1$ for $\Gamma^{0}(2)$ [an overall factor of 2 multiplying Eq. (8) does not change Fig. 3, indeed Eq. (8) is only derived in Ref. 5 up to an overall positive constant].

Figure 2 shows how the fixed point $\sigma_{*}=\frac{1+i}{2}$ of $\Gamma_{0}(2)$ moves as $z$ is varied: particle-hole symmetry constrains it to keep to the real curve of $\lambda$ shown in Fig. 1. The point associated with $z=1$ can be gained from $z=-2$ either by going anticlockwise or clockwise. Suppose first that $z$ increases monotonically between -2 and 1 as the Zeeman splitting is varied smoothly from well-split spins, $\Gamma_{0}(2)$, to degenerate spins, $\Gamma^{0}(2)$. Figure 2 shows that, as $z$ is increased from -2 to -1 , the fixed point at $\sigma_{*}=\frac{1+i}{2}$ of the $\Gamma_{0}$ (2) flow (Fig. 3) follows the semicircular arc of radius $\frac{1}{2}$, moving down to the left until it hits the origin in the $\sigma$ plane, $\sigma_{*}=0$ when $z=-1$. It then continues up the imaginary axis,

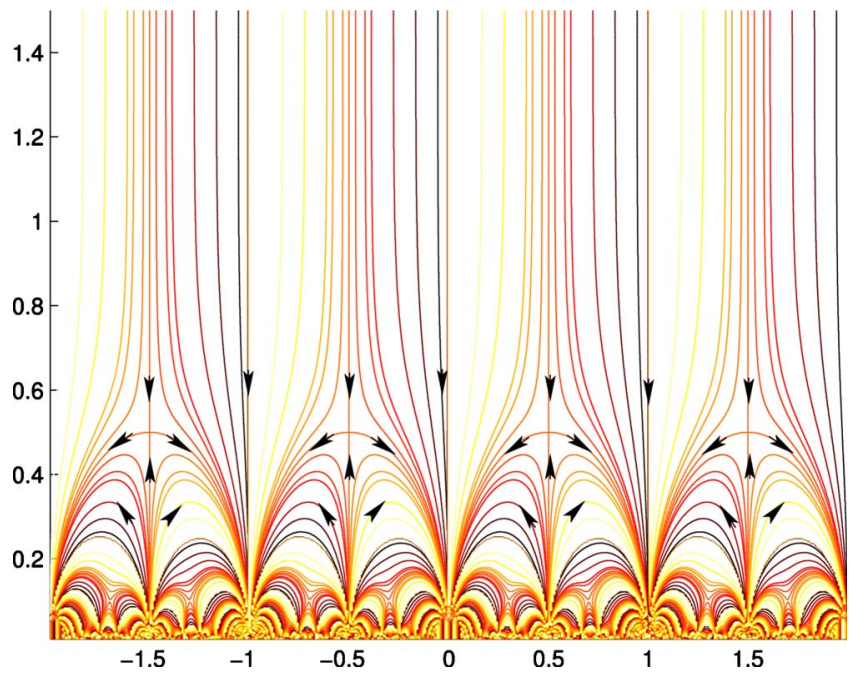

FIG. 3. (Color online) Spins well split, $\Gamma_{0}(2)$ symmetry.

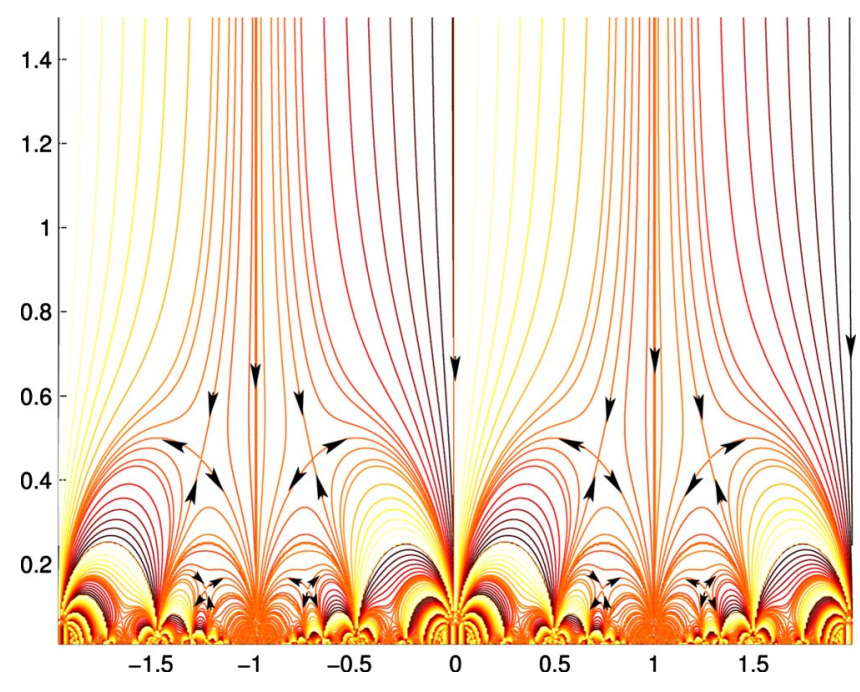

FIG. 4. (Color online) $z=-10$.

through $\sigma_{*}=i$ for $z=-\frac{1}{2}$ to $\sigma_{*}=i \infty$ for $z=0$ [near $z=0$, Eq. (12) can be replaced with

$$
\Sigma_{s}(\sigma)=\frac{1}{i \pi} \frac{\tilde{z}}{\left(\widetilde{z} \vartheta_{2}^{4}+\vartheta_{3}^{4}\right)},
$$

where $\tilde{z}=1 / z$, multiplying $\Sigma_{s}$ by a constant does not change the topology of the flow]. The fixed point subsequently moves down from $\sigma_{*}=1+i \infty$ to $\sigma_{*}=1+i\left[\mathrm{a} \Gamma^{0}(2)\right.$ fixed point, Fig. 11] as $z$ increases from 0 to 1 . While experimental data to date do show a fixed point on the semicircle spanning 1 to 0 that is to the left of $\frac{1+i}{2}, 9,10,24$ it does not seem likely that this sequence of flows can be the correct one. A fixed point at $\sigma_{*}=i$ when $z=-\frac{1}{2}$ has never been seen in any Hall sample. Indeed such fixed points were identified in Ref. 17 as being associated with bosonic pseudoparticles excitations (as in the superconductor-insulator transition of Ref. 16, for example), rather the fermionic pseudoparticle excitations of the quantum Hall effect. Since no quantum Hall sample to date has

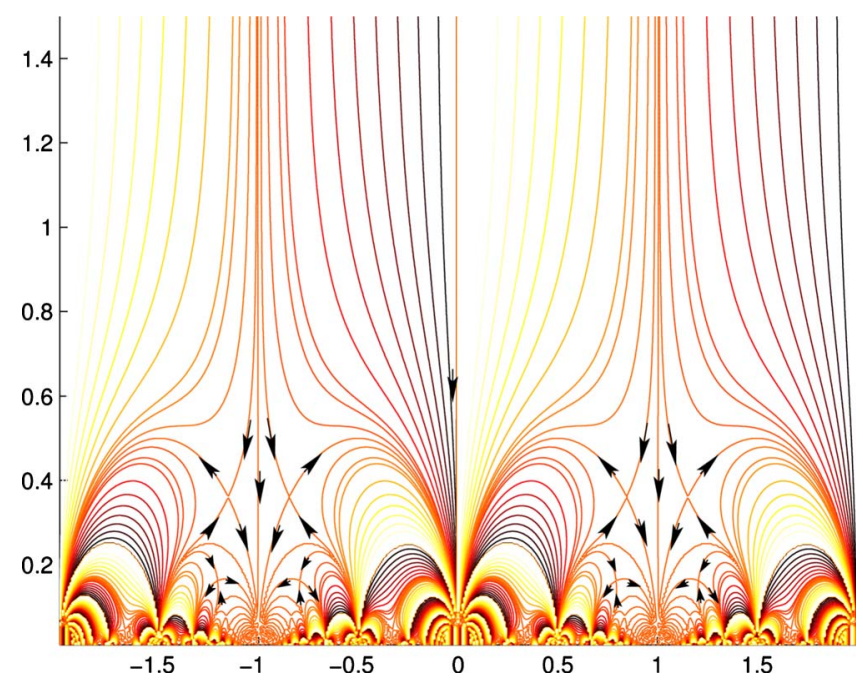

FIG. 5. (Color online) $z=-100$. 


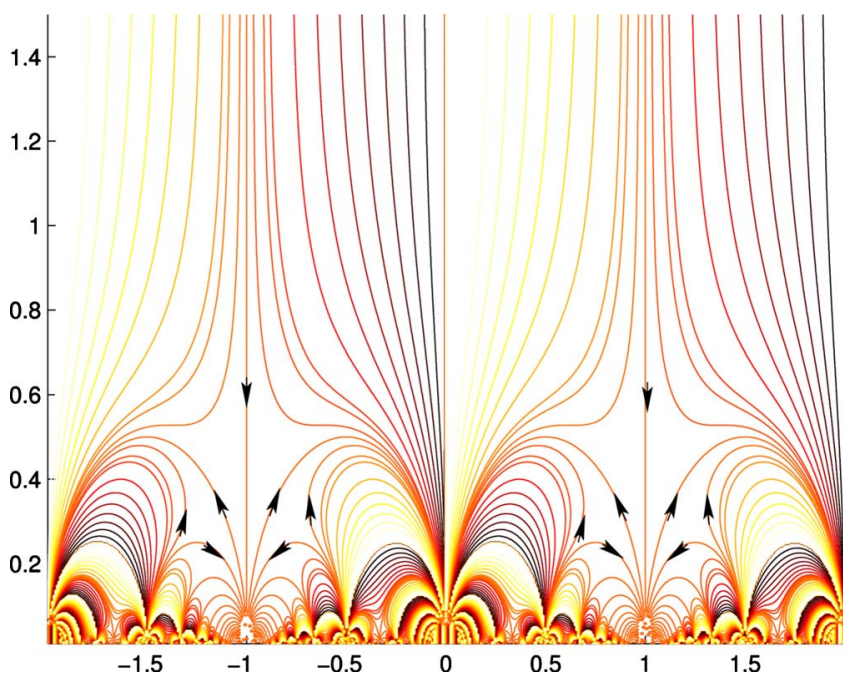

FIG. 6. (Color online) $|z|=\infty$.

ever exhibited a critical point at $\sigma=i$ we exclude this possibility.

An alternative possibility is that the flow morphs from Figs. $3-11$ by decreasing $z$ from -2 going through $-\infty$ to $+\infty$ to continue down to +1 . The nine plots in Figs. $3-11$ show the series of flows for

$$
z=-2,-10,-100, \pm \infty, 100,20,10,2 \text {, and } 1 \text {. }
$$

The fixed point at $\frac{3+i}{2}$ for samples with well-split spins moves left and down as the Zeeman splitting is decreased, along the semicircle of radius $\frac{1}{2}$ centered $\sigma=\frac{3}{2}$, until it hits the real axis at $\sigma=1$ (for $|z|=\infty$ ), where it merges with the incoming fixed point coming from $\frac{1+i}{2}$ on the left. It then moves vertically upwards to the point $1+i$ when $z=1$, which is the fixed point for degenerate samples with symmetry $\Gamma^{0}(2)$. Every flow in the sequence has $\Gamma(2)$ symmetry, which is enhanced to $\Gamma_{0}(2)$ or $\Gamma^{0}(2)$ at the extreme values $z=-2$ and $z=1$, respectively.

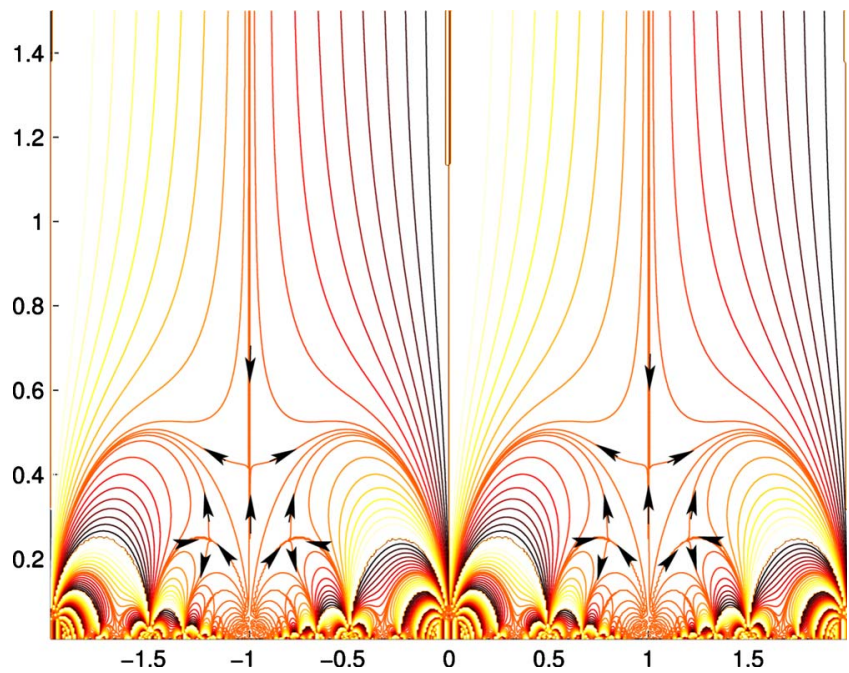

FIG. 7. (Color online) $z=100$.

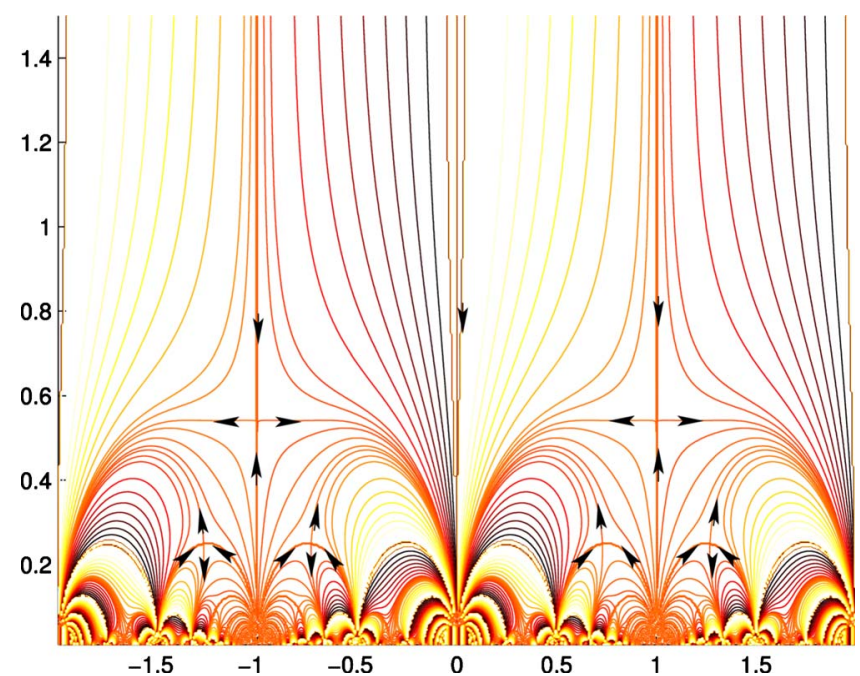

FIG. 8. (Color online) $z=20$.

In real samples particle-hole symmetry is hardly likely to be an exact symmetry of the system, there will be deviations from this picture. But any deviations will be small if particlehole interchange is a reasonably good symmetry: for example, if $\frac{m_{p}-m_{h}}{m_{p}+m_{h}}$ is small, where $m_{p}$ is the particle mass and $m_{h}$ the hole mass. In particular, the collision of the critical points at $\sigma=1(|z|=\infty)$ seems likely to be an artifact of the mathematical idealization of exact particle-hole symmetry, as there is no obvious physical mechanism governing the merging of two critical points as the Zeeman energy is reduced. The most plausible scenario here is that the merge is postponed in a real sample until the Zeeman splitting is reduced to very small values and the proposed trajectory of a real sample, in which particle-hole interchange is a good but not exact symmetry, is shown in Fig. 12. This is a small perturbation of the mathematically idealized flows shown in Figs. 3-11 which is still compatible with the proposed symmetries.

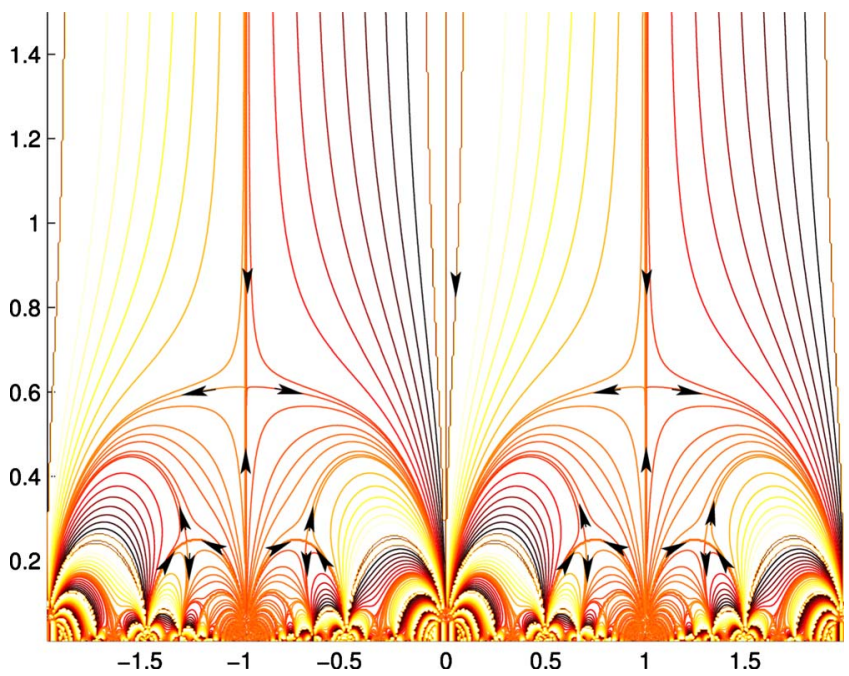

FIG. 9. (Color online) $z=10$. 


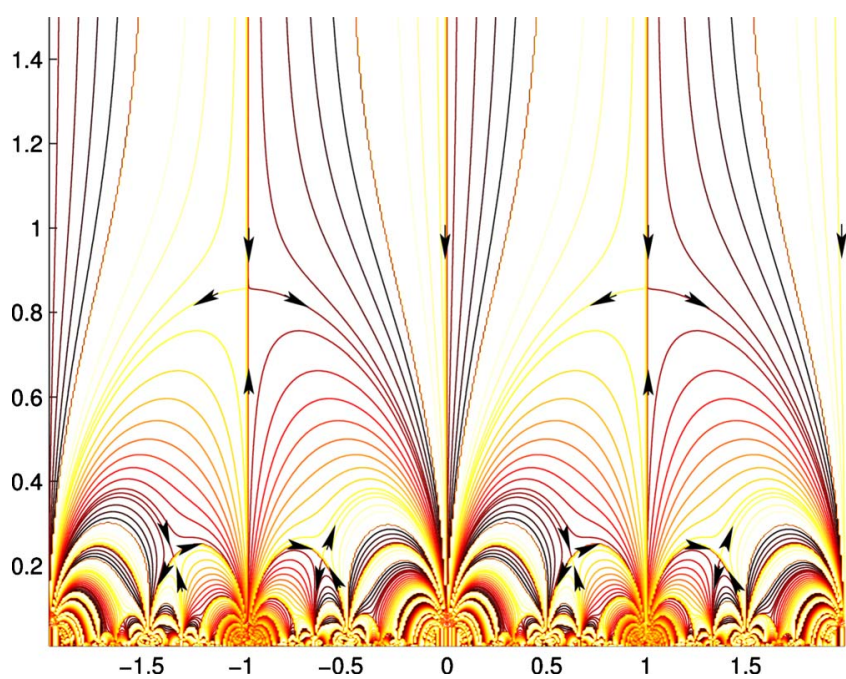

FIG. 10. (Color online) $z=2$.

\section{CONCLUSIONS}

The topology of the temperature flow of conductivities in quantum Hall samples is tightly constrained by the law of corresponding states, expressed in terms of modular transformations on the complex conductivity [Eq. (2)]. For the extreme cases of well-split spins and degenerate spins the critical points in the complex plane are determined by the symmetry. For intermediate values of the Zeeman splitting modular symmetry does not determine the position of the critical points but one can assume that they move around the complex plane in a continuous manner as the Zeeman splitting is varied.

In the case of samples exhibiting particle-hole symmetry, modular symmetry is particularly powerful, leading to the statement that the curves in Fig. 1, and their images under $\Gamma(2)$ modular transformations will be trajectories of the conductivity flow as the temperature is varied keeping the magnetic field fixed. This statement should be true for any Zeeman splitting. Zeeman energies which are large

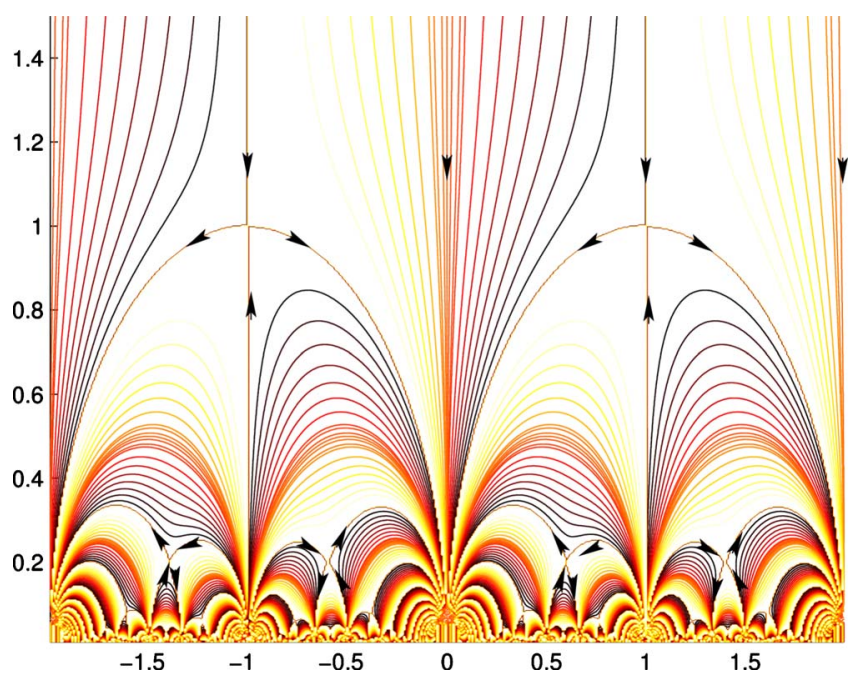

FIG. 11. (Color online) Spins degenerate, $\Gamma^{0}(2)$ symmetry.

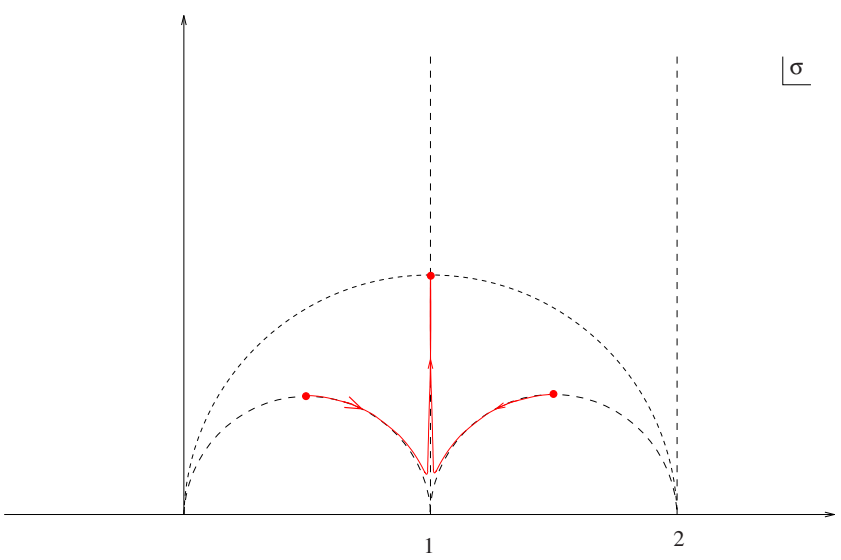

FIG. 12. (Color online) Proposed movement of the fixed point $\sigma=\frac{(1+i)}{2}$ for nondegenerate spins, $\Gamma_{0}(2)$, to $\sigma=1+i$ for spindegenerate samples, $\Gamma^{0}(2)$, as the Zeeman energy is reduced.

enough to give well-split spins result in critical points at $\sigma_{x y}+i \sigma_{x x}=n+\frac{1+i}{2}$ between integer Hall plateaux $\sigma_{x y}=n$ and $n+1$ (Fig. 3). Zeeman energies which are so small that the spins are degenerate give critical points at $\sigma_{x y}+i \sigma_{x x}=2 n+1+i$ between integer Hall plateaux $\sigma_{x y}=2 n$ and $2 n+2$ (Fig. 11).

The form of the flow as the Zeeman splitting is varied from the spin split to the spin-degenerate case is shown in Figs. 3-11. Figure 12 shows proposed trajectories of two critical points as the Zeeman energy is reduced in a real sample exhibiting symmetry under particle interchange which is good but not exact.

The analysis here has assumed that the parameter $z$ varies monotonically as the Zeeman energy is varied, implying that the critical point at $\sigma=\frac{1+i}{2}$ in the transition between $\sigma=1$ and $\sigma=0$ in spin-split samples moves to the right, down toward to $\sigma=1$, as the Zeeman splitting is reduced. There is as yet no experimental evidence for such behavior: indeed in Refs. 9,10 , and 24 a critical point is found on the semicircle spanning 0 to 1 in the complex conductivity plane which is to the left of $\sigma=\frac{1+i}{2}$. This could be a consequence of a constant rescaling of $\sigma_{x x},{ }^{11}$ or, perhaps more likely, it may indicate

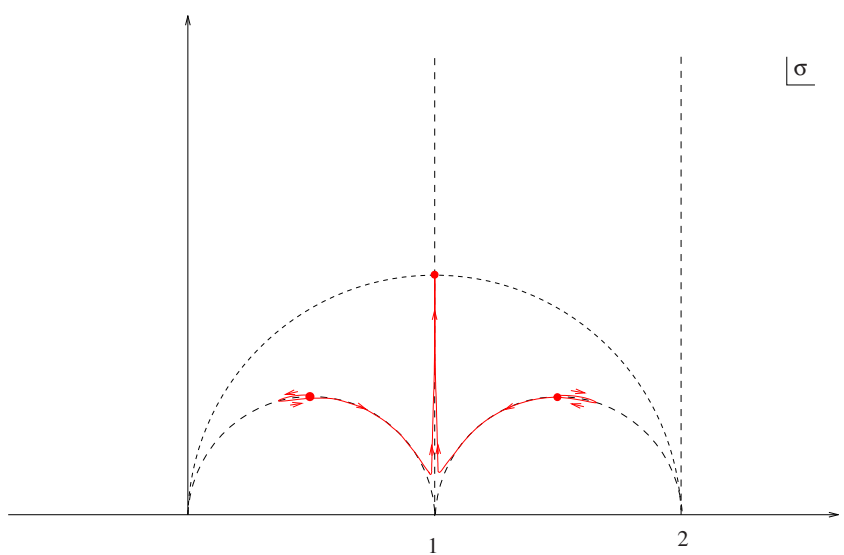

FIG. 13. (Color online) Possible movement of the fixed points if the mathematical parameter $z$ does not vary monotonically with the Zeeman splitting. 
that $z$ is not monotonic. It could be that the critical point at $\sigma=\frac{1+i}{2}[z=-2$ in Eq. (12)] in spin-split samples first starts to move down and to the left $(z>-2)$ and then reverses to retrace its steps back to $z=-2$ before starting to travel down to the right toward $\sigma=1$ as $z$ decreases below -2 . A possible trajectory is shown in Fig. 13. While this would be compatible with the experimental data to date any physical explanation of such a trajectory, which is certainly allowed by the law of corresponding states combined with particle-hole symmetry, would go beyond the general predictions following from these assumptions and would probably require a more specific microscopic model. More experimental data would be welcome in order to determine the true behavior of the critical points.

\section{ACKNOWLEDGMENTS}

B.P.D. acknowledges the warm hospitality of Pei-Ming Ho and Chi-Te Liang during his visit funded by the National Science Council, Taiwan, ROC (Grant No. NSC 098-2912I-002-097). It is a pleasure to thank Pei-Ming Ho and Chi-Te Liang for discussions. *bdolan@thphys.nuim.ie

${ }^{1}$ A. Shapere and F. Wilczek, Nucl. Phys. B 320, 669 (1989).

${ }^{2}$ S. Kivelson, D.-H. Lee, and S.-C. Zhang, Phys. Rev. B 46, 2223 (1992).

${ }^{3}$ C. A. Lütken and G. G. Ross, Phys. Rev. B 45, 11837 (1992); 48, 2500 (1993).

${ }^{4}$ B. P. Dolan, J. Phys. A 32, L243 (1999).

${ }^{5}$ B. P. Dolan, Nucl. Phys. B 554, 487 (1999).

${ }^{6}$ S. S. Murzin, M. Weiss, A. G. M. Jansen, and K. Eberl, Phys. Rev. B 66, 233314 (2002).

${ }^{7}$ S. S. Murzin, S. I. Dorozhkin, D. K. Maude, and A. G. M. Jansen, Phys. Rev. B 72, 195317 (2005).

${ }^{8}$ C. F. Huang, Y. H. Chang, H. H. Cheng, C.-T. Liang, and G. J. Hwang, Physica E 22, 232 (2004).

${ }^{9}$ D. Hang, R. Dunford, G. Kim, H. Yeh, C. Huang, D. Ritchie, I. Farrer, Y. Zhang, C. Liang, and Y. Chang, Microelectron. J. 36, 469 (2005).

${ }^{10}$ C. F. Huang, Y. H. Chang, H. H. Cheng, Z. P. Yang, H. D. Yeh, C. H. Hsu, C.-T. Liang, D. R. Hang, and H. H. Lin, J. Phys.: Condens. Matter 19, 026205 (2007).

${ }^{11}$ B. P. Dolan, Phys. Rev. B 62, 10278 (2000).

${ }^{12}$ S.-C. Zhang, T. H. Hansson, and S. Kivelson, Phys. Rev. Lett. 62, 82 (1989).

${ }^{13}$ C. P. Burgess and C. A. Lutken, Nucl. Phys. B 500, 367 (1997);
Phys. Lett. B 451, 365 (1999).

${ }^{14}$ J. K. Jain, Phys. Rev. Lett. 63, 199 (1989); Adv. Phys. 41, 105 (1992).

${ }^{15}$ N. Koblitz, Introduction to Elliptic Curves and Modular Forms, Graduate Texts in Mathematics Vol. 94 (Springer, New York, 1984).

${ }^{16}$ M. P. A. Fisher, Phys. Rev. Lett. 65, 923 (1990).

${ }^{17}$ C. P. Burgess and B. P. Dolan, Phys. Rev. B 63, 155309 (2001).

${ }^{18}$ B. P. Dolan, Symmetry, Integr. Geom.: Methods Appl. (SIGMA), 3, 010 (2007).

${ }^{19}$ C. P. Herzog, P. Kovtun, S. Sachdev, and D. T. Son, Phys. Rev. D 75, 085020 (2007).

${ }^{20}$ H. P. Wei, D. C. Tsui, M. A. Paalanen, and A. M. M. Pruisken, Phys. Rev. Lett. 61, 1294 (1988); A. M. M. Pruisken, ibid. 61, 1297 (1988).

${ }^{21}$ Wanli Li, G. A. Csáthy, D. C. Tsui, L. N. Pfeiffer, and K. W. West, Phys. Rev. Lett. 94, 206807 (2005).

${ }^{22}$ C. P. Burgess, R. Dib, and B. P. Dolan, Phys. Rev. B 62, 15359 (2000).

${ }^{23}$ E. T. Whittaker and G. N. Watson, A Course of Modern Analysis, 4th ed. (Cambridge University Press, Cambridge, London, 1927).

${ }^{24}$ M. Hilke, D. Shahar, S. Song, D. Tsui, Y. Xie, and M. Shayegan, Europhys. Lett. 46, 775 (1999). 